\title{
IRRADIATION FOR THE SAFETY AND QUALITY OF HOME STYLE FROZEN SNACKS
}

\author{
Z. Irawati ${ }^{1}$, L. Natalia ${ }^{2}$, C.M. Nurcahya ${ }^{1}$, F. Anas $^{1}$ \\ and M. Tampubolon ${ }^{3}$ \\ ${ }^{1}$ Centre for Research and Development of Isotopes and Radiation Technology, \\ National Nucelar Energy Agency, Jl. Cinere Pasar Jumat PO Box 7002, JKSKL, \\ Jakarta 12070 Indonesia. \\ ${ }^{2}$ Research Institute for Veterinary Science, Jl. Marthadinata 52 PO Box 16114, Bogor West Java. \\ ${ }^{3}$ Pharmacy Division, Faculty of Natural Science and Mathematics, \\ National Institute for Science and Technology, \\ Srengseng Sawah, Jakarta, Indonesia.
}

\begin{abstract}
IRRADIATION FOR THE SAFETY AND QUALITY OF HOME STYLE FROZEN SNACKS. There are to market frozen prepared meals for microwaving or conventional way of preparation. Such meals. which offer convenience growing trends in Indonesia, and lesser time preparation are available in the developed countries, either chilled with limited shelf-life or frozen for long term sale in supermarkets. Irradiation at pasteurization doses has a potential role in improving the microbiological safety and shelf-life of chilledprepared meals. Medium doses of gamma irradiation, 3-7 kGy, applied to spring rolls, rissole and croquette, in combination with frozen state during irradiation, followed by storage at $5 \pm$ $2^{\circ} \mathrm{C}$, have been conducted in order to ensure the safety and quality of the products during storage. The three different snacks. six pieces each. were vacuum packed in a laminate pouch of Poliester/Aluminum foil/LLDPE followed by fteezing over night at $-20^{\circ} \mathrm{C}$ prior to irradiation at cryogenic condition. Irradiation at a dose of $7 \mathrm{kGy}$ could relatively maintain the quality of the products during three months of storage $5 \pm 2{ }^{\circ} \mathrm{C}$, based on different parameters, such as microbiological assessments, some chemical characteristics, and organoleptic analysis using five hedonic scales.
\end{abstract}

Key words : gamma irradiation, frozen prepared meals, laminate pouch.

\section{INTRODUCTION}

In Indonesia, there are growing trends to market frozen prepared meals as convenience foods, intended for microwaving or conventional way of preparation. The social changes such as the growing of working mothers, the attraction to outdoor activities, growing income but no interest in cooking. might contribute to the need of reduced time for the preparation and consumption of a complete meal. Furthemore, in promoting the prepared meats, the focus should be kept on the pleasing the potential consumers. The convenience food business indicates a large potential for development and it is shown from the report that total sold chilled food in the world was US $\$ 128.2$ billion in 2002 [1].

Application of irradiation technology at medium doses, in combination with cryogenic condition along the process, for some ethnic home style frozen foods as savoury products have been conducted to reduce the freezer dependency, and to accelerate safety and shelf-life $[2,3,4]$. The storage time 
up to three months at $4{ }^{\circ} \mathrm{C}$ did not show any significant effect on the quality of the products [2]. In the advanced and developing countries, such meals, which offer convenient and less time for preparation, are marketed either under chilled for limited shelf-life, or frozen for long term sale in supermarkets. Spring rolls, with tradition name lumpia, rissole and croquette are ethnic foods which often fteshly prepared and marketed on a day to day basis at ambient condition in most tropical countries. These products donot need defrost, but directly deep fried in hot cooking oil at about $175-200^{\circ} \mathrm{C}$ for five minutes until golden brown colour appears. Spring rolls are usually served as snack, together with vallous type of sauce and fresh chili.

It is realized that irradiation has a potential role in improving the microbiological safety and extending the shelf-life of chilled-prepared meals. The quality of such meals, currently marketed under frozen condition. could possibly be improved through irradiation under chilled storage to ensure not only microbiologically safe but also sufficient shelf-life to meet market requirement resulting in saving energy and cost.

Objective of this study is to evaluate the effectiveness of iradiation as a treatment to ensure microbiological safety and extend the shelf-life of the home style frozen snacks which are kept under chilled temperatures for long term.

\section{MATERIALS AND METHODS}

\section{Materials}

Commercial frozen spring rolls or lumpia, rissole and croquette without preservatives were purchased from home and food industries in Jakarta respectively. Composition of the products are presented at Table 1 and 2. A laminate pouch of Polyester / Aluminum foil/LLDPE as packaging material was selected in the research work.

\section{Methods and Sample Preparations}

Filler of spring roll was prepared according to the following steps: sliced of three pieces shallot and four slices garlic were separately fried in vegetable oil until smell fragrance comes out The rest of filler materials. as listed in Table 1, were added, and mixed thoroughly, decanted, then split into 20 portions for further preparation. The final step of spring rolls was prepared by spreading the cooked filler on top of a spring roll sheet, fold it into rectangular shape, then sticked the edge with a drop of stirred egg as glue.

Sweet and tauco sauce were prepared siparately by mixing all materials as listed in Table 1, then continued to boil in order to cook and raise the consistency. The sauce was not irradiated but sarved separately. 
Rissole sheet was prepared by pouring the materials into a bowl, whisked and mixed thoroughly. The mixing materials then filed in a pan and make into a very tiny sheet. Rissole filler was cooked as the sauce methods as making to spring rolls but the materials used in this preparation is different as listed in Table 2. Filler was poured onto a piece of sheet, fold it similar to an envelope shape, then sticked the edge with a drop of stirred egg as glue. Finally the risoles was rolled on the spreaded bread crumbs until the surtace was totally covered with it.

Mash potatoes together with ingredients were prepared as a croquette wrapper. The rest step in making croquette was the same as in the rissole making until the final preparation was made.

\section{Packaging Material}

Selected packaging material as prepackaged foods was a laminate pouch of PET $12 \mu \mathrm{m} / \mathrm{LDPE}$ as adh. $2 \mu \mathrm{m} / \mathrm{Al}$-foil $7 \mu \mathrm{m} / \mathrm{LDPE}$ as adh./LIDPE $50 \mu \mathrm{m}$ as used in the previous work [1-2]. Each type of snacks, 6 pieces of each type then placed into a pouch, was packed under vacuum in a laminate flexible packaging film. The samples were conditioned for 24 hours at $-18^{\circ} \mathrm{C}$ then removed to a styrofoam boxes $\left(33.75 \times 36.25 \times 51.25 \mathrm{~cm}^{3}\right)$ filled with dry ice and hermetically sealed prior to irradiation.

\section{Irradiation treatment}

Gamma irradiation was conducted at IRKA irradiator at National Nuclear Energy Agency, Pasar Jumat, Jakarta. Cobalt-60 was used as the source of ionizing radiation at the dose rate of $7.65 \mathrm{kGy} / \mathrm{h}$. Samples were irradiated in a Gamma lrradiator with doses of 3, 5, and $7 \mathrm{kGy}$ respectively.

\section{Storage}

Both treated and untreated samples were kept at chilled temperatures $\left(3-7^{\circ} \mathrm{C}\right)$ in order to determine the effect of gamma irradition on the microbiological load using different parameters as well as physico-chemical charactetistics and sensoric evaluation would also be carried out Evaluation will be conducted each month for total storage duration of about 3 months. 
Table 1. Ingredients, for making 20 pieces of spring rolls.

\begin{tabular}{|c|c|}
\hline Materials / Ingredients & Quantity \\
\hline \multicolumn{2}{|l|}{ Spring rolls and filler: } \\
\hline ready prepared-spring rolls sheets & 20 pieces \\
\hline Minced-beef/chicken meat & $250 \mathrm{~g}$ \\
\hline Peeled shrimp & $100 \mathrm{~g}$ \\
\hline Small cut of can/boiled young bamboo shoot & $300 \mathrm{~g}$ \\
\hline Fine sliced-shallot & 3 pieces \\
\hline Fine sliced-garlic & 4 pieces \\
\hline Salt & 1 tea spoon \\
\hline Diluted sago flour as glue & $1 / 2$ spoon \\
\hline Sweet soya sauce & 1 spoon \\
\hline \multicolumn{2}{|l|}{ Sweet sour sauce } \\
\hline Sago flour & $50 \mathrm{~g}$ \\
\hline Sliced-palm sugar & $50 \mathrm{~g}$ \\
\hline Sweet soya sauce & $1 / 2$ spoon \\
\hline Water & $150 \mathrm{cc}$ \\
\hline \multicolumn{2}{|l|}{ Tauco sauce } \\
\hline Tauco & 4 spoons \\
\hline Minced garlic & 2 tea spoons \\
\hline Chili & 5 pieces \\
\hline Sliced palm sugar & 3 spoons \\
\hline Fine salt & $1 / 2$ tea spoon \\
\hline Venegar & $1 \frac{1}{2}$ tea spoon \\
\hline Sweet soya & 1 spoon \\
\hline water & $200 \mathrm{cc}$ \\
\hline \multicolumn{2}{|l|}{ Cucumber pickle } \\
\hline Seedless cucumber & 2 pieces \\
\hline Fine salt & 1 tea spoon \\
\hline Sugar & 2 spoons \\
\hline Vinegar & $1 / 2$ tea spoon \\
\hline
\end{tabular}

Table 2. Ingredients, for making 10-20 pieces of rissole and 8-12 pieces of croquette.

\begin{tabular}{|l|c|c|}
\hline \multicolumn{1}{|c|}{ Materials/ Ingredients } & Quantity & Quantity \\
\hline Margaine & & Croquette filler \\
\hline Minced- beef/chicken meat & 2 spoons & 2 spoons \\
\hline Wheat flour & $50 \mathrm{~g}$ & $150 \mathrm{~g}$ \\
\hline Chicken broth & 2 spoons & $100 \mathrm{ml}$ \\
\hline Shallot & $100 \mathrm{ml}$ & 5 pieces \\
\hline Garlic & 5 pieces & - \\
\hline Green Beans & 4 slices & - \\
\hline Carrot & $50 \mathrm{~g}$ & 1 piece \\
\hline Sugar pease (legume) & - & 20 pieces \\
\hline Cheese & - & - \\
\hline Ground peppers, salt & $100 \mathrm{~g}$ & - \\
\hline Eggs & 1 ten spoons & - \\
\hline & 2 & Croquette wrapper \\
\hline Milk & $250 \mathrm{ml}$ & - \\
\hline Eggs & 2 & 2 \\
\hline Wheat flour & $20 \mathrm{~g}$ & - \\
\hline Salt & $1 \mathrm{tea} \mathrm{spoon}$ & - \\
\hline Steamed potatoes & - & $500 \mathrm{~g}$ \\
\hline Chicken broth & - & $100 \mathrm{ml}$ \\
\hline Ground nutmeg & $-100 \mathrm{~g}$ & $100 \mathrm{~g}$ \\
\hline Bread crumbs & wrapper & \\
\hline
\end{tabular}

4 


\section{Methods of analysis}

\section{Microbiological assessments}

The microbiological assessments were conducted at each step of the preparation to determine the microbiological load of raw and semi-cooked materials used in making the different types of snacks. The methods used in the microbilogical assessments were Total Plate Count (TPC). Total Mould and Yeast Count (TMYC), Coliform, E.coli, Salmonella spp., Staphylococcus spp., and Clostridium perfringens.

\section{Water activity (Aw), pH, moisture, carbohydrate, fat and protein contents}

Water activity was measured using a Shibaura Aw meter WA-360, at $28-30^{\circ} \mathrm{C}$. The $\mathrm{pH}$ of three different type of snacks were measured using a Microcomputer $\mathrm{pH}$ meter LEC 60. Moisture content was measured using gravimetry. Carbohydrate content was analyzed according to Luff-Schroll method of analysis. Fat content was determined by extraction the samples using petroleum benzene $\left(60-80^{\circ} \mathrm{C}\right)$ for $5 \mathrm{~h}$. The physicochemical characterizations were only conducted before and after 3 months of storage while the microbiological assessments of each sample were done along the storage time at $5 \pm 2^{\circ} \mathrm{C}$.

\section{Sensory evaluation}

Sensory evaluation was done by 10 to 20 selected panellists using a 5 point hedonic scale. A score of $5=$ excellent, $4=$ good, $3=$ fair, $2=$ poor, and 1 = extreemely poor.

\section{RESULTS AND DISCUSSION}

Results of microbial assessments on raw-mix vegetables, uncooked seasonings and bread crumb, are presented in Table 3. The assessments is a mandatory for food industry to implement Hazard Analytical Critical Control Point (HACCP) within the processing steps [5]. Clostridium prefringens were not found in the analyzed samples. 
Table 3. Microbial load (in cfu/g) * in raw mix-vegetables and uncooked seasonings of spring rolls, rissole and croquette, before preparation.

\begin{tabular}{|l|c|c|c|c|c|}
\hline \multirow{2}{*}{ Microbes } & \multicolumn{5}{|c|}{ Type of product } \\
\cline { 2 - 6 } & $\begin{array}{c}\text { Raw-mix- } \\
\text { vegetables }\end{array}$ & $\begin{array}{c}\text { Spring rolls } \\
\text { filler }\end{array}$ & $\begin{array}{c}\text { Rissole } \\
\text { filler }\end{array}$ & $\begin{array}{c}\text { Croquett } \\
\text { filler }\end{array}$ & $\begin{array}{c}\text { Bread } \\
\text { Crumbs }\end{array}$ \\
\hline TPC & $1.2 \times 10^{4}$ & $1.9 \times 10^{3}$ & $3.3 \times 10^{4}$ & $3.1 \times 10^{4}$ & $1.7 \times 10^{3}$ \\
\hline TMYC & $1.1 \times 10^{5}$ & $1.3 \times 10^{5}$ & $1.2 \times 10^{4}$ & $1.3 \times 10^{3}$ & $1.2 \times 10^{3}$ \\
\hline $\begin{array}{l}\text { E.coli } \\
\text { Coliform }\end{array}$ & negative & negative & negative & negative & negative \\
\cline { 2 - 6 } $\begin{array}{l}\text { Pseudomonas } \\
\text { spp. } \\
\begin{array}{l}\text { Slaphylococcus } \\
\text { spp }\end{array}\end{array}$ & 0 & negative & negative & negative & negative \\
\cline { 2 - 6 } $\begin{array}{l}\text { Clostridium } \\
\text { perfringens }\end{array}$ & negative & negative & negative & negative & negative \\
\hline
\end{tabular}

*Average of three replications

There was no microbial growth found neither in cooked filler nor in cooked vegetables used in the preparation of making the three different snacks. Results on microbial assessment of spring rolls is presented in Table 4. It showed that this type of food could only be stored at $5 \pm 2^{\circ} \mathrm{C}$ for about one month. After one month of storage, both control and irradiated samples up to $5 \mathrm{kGy}$, did not show promising results, because the filler became juicy and produced some gases. The gases with strong bad smell infiltrated the package through tiny pinholes in the sealed area during storage period. It was probably a result of fermentation due to biochemistry process of bamboo shoot which used as filler material during preparation of making spring rolls. Kitchen utilities also play an important role in the increasing of initial microbial loads. Priadi et.al. [6] concluded that microbiological safety and quality of chicken and chicken products collected from local market were poor and potential sources of foodborne disease outbreak in Indonesia. Ashari et.al.[7] had similar conclusion for the meatball consumption regarding its safety and convenience.

Table 5 and Table 6 show the results of unirradiated and irradiated rissole and croquette during storage at $5 \pm 2{ }^{\circ} \mathrm{C}$ respectively. It is indicated that rissole and croquette showed better result than spring rolls. It is also indicated from the results that gamma irradiation with the dose of 5-7 kGy could eliminate all type of microbes observed in the samples. However, initial contamination of bread crumbs was really high. It is suggested that the crumbs should be irradiated at medium dose prior to use. 
Table 4. Microbial load (in cfu/g) * of unirradiated and irradiated spring rolls with doses up to $7 \mathrm{kGy}$ during three months of storage at $5 \pm 2{ }^{\circ} \mathrm{C}$.

\begin{tabular}{|c|c|c|c|c|c|}
\hline \multirow[t]{2}{*}{ Microbes } & \multirow{2}{*}{$\begin{array}{l}\text { Stroge time } \\
\text { (month) }\end{array}$} & \multicolumn{4}{|c|}{ Irradiation dose (kGy) } \\
\hline & & 0 & 3 & 5 & 7 \\
\hline \multirow[t]{3}{*}{ TPC } & 0 & $2.30 \times 10^{3}$ & 0 & 0 & 0 \\
\hline & 1 & $16.30 \times 10^{3}$ & 0 & 0 & 0 \\
\hline & 3 & $>10^{3}$ & $4.18 \times 10^{3}$ & $4.50 \times 10^{3}$ & 0 \\
\hline \multirow[t]{3}{*}{ TMYC } & 0 & 0 & 0 & 0 & 0 \\
\hline & 1 & $1.42 \times 10^{3}$ & $1.20 \times 10^{3}$ & 0 & 0 \\
\hline & 3 & $>10^{3}$ & $4.15 \times 10^{2}$ & $2.76 \times 10^{2}$ & 0 \\
\hline \multirow[t]{3}{*}{ E.coli } & 0 & negative & negative & negative & negative \\
\hline & 1 & negative & negative & negative & negative \\
\hline & 3 & negative & negative & negative & negative \\
\hline \multirow[t]{3}{*}{ Coliform } & 0 & negative & negative & negative & negative \\
\hline & 1 & negative & negative & negative & negative \\
\hline & 3 & negative & negative & negative & negative \\
\hline \multirow{3}{*}{$\begin{array}{l}\text { Pseudomonas } \\
\text { spp. }\end{array}$} & 0 & 0 & 0 & 0 & 0 \\
\hline & 1 & 0 & 0 & 0 & 0 \\
\hline & 3 & 0 & 0 & 0 & 0 \\
\hline \multirow{3}{*}{$\begin{array}{l}\text { Slaphylococcus } \\
\text { spp }\end{array}$} & 0 & negative & negative & negative & negative \\
\hline & 1 & negative & negative & negative & negative \\
\hline & 3 & $>10^{3}$ & $>10^{2}$ & 10 & negative \\
\hline \multirow{3}{*}{$\begin{array}{l}\text { Clostridium } \\
\text { perfringens }\end{array}$} & 0 & negative & negative & negative & negative \\
\hline & 1 & negative & negative & negative & negative \\
\hline & 3 & negative & negative & negative & negative \\
\hline
\end{tabular}

*Average of three replications

Table 5. Microbial load*(in cfu/g) of unirradiated and irradiated rissole with doses up to $7 \mathrm{kGy}$ during three months of storage at $5 \pm 2^{\circ} \mathrm{C}$.

\begin{tabular}{|c|c|c|c|c|c|}
\hline \multirow[t]{2}{*}{ Microbes } & \multirow{2}{*}{$\begin{array}{c}\text { Stroge } \\
\text { time } \\
\text { (month) }\end{array}$} & \multicolumn{4}{|c|}{ Irradiation dose (kGy) } \\
\hline & & 0 & 3 & 5 & 7 \\
\hline \multirow[t]{3}{*}{ TPC } & 0 & $2.71 \times 10^{3}$ & 0 & 0 & 0 \\
\hline & 1 & $3.98 \times 10^{3}$ & 0 & 0 & 0 \\
\hline & 3 & $>10^{3}$ & $4.37 \times 10^{2}$ & $2.30 \times 10$ & 0 \\
\hline \multirow[t]{3}{*}{ TMYC } & 0 & $3.60 \times 10^{3}$ & 0 & 0 & 0 \\
\hline & 1 & $3.90 \times 10$ & $1.40 \times 10$ & 0 & 0 \\
\hline & 3 & $>10^{3}$ & $4.10 \times 10$ & $1.20 \times 10$ & 0 \\
\hline \multirow[t]{3}{*}{ E.coli } & 0 & negative & negative & negative & negative \\
\hline & 1 & negative & negative & negative & negative \\
\hline & 3 & negative & negative & negative & negative \\
\hline \multirow[t]{3}{*}{ Coliform } & 0 & negative & negative & negative & negative \\
\hline & 1 & negative & negative & negative & negative \\
\hline & 3 & negative & negative & negative & negative \\
\hline \multirow{3}{*}{$\begin{array}{l}\text { Pseudomonas } \\
\text { spp. }\end{array}$} & 0 & 0 & 0 & 0 & 0 \\
\hline & 1 & 0 & 0 & 0 & 0 \\
\hline & 3 & 0 & 0 & 0 & 0 \\
\hline \multirow{3}{*}{$\begin{array}{l}\text { Slaphylococcus } \\
\text { spp }\end{array}$} & 0 & negative & negative & negative & negative \\
\hline & 1 & negative & negative & negative & negative \\
\hline & 3 & $>10^{3}$ & $>10^{2}$ & 10 & negative \\
\hline \multirow{3}{*}{$\begin{array}{l}\text { Clostridium } \\
\text { perfringens }\end{array}$} & 0 & negative & negative & negative & negative \\
\hline & 1 & negative & negative & negative & negative \\
\hline & 3 & negative & negative & negative & negative \\
\hline
\end{tabular}

*Average of three replications 
Table 6. Microbial load*(in cfu/g) of unirradiated and irradiated croquette with doses up to $7 \mathrm{kGy}$ during three months of storage at $5 \pm 2{ }^{\circ} \mathrm{C}$.

\begin{tabular}{|c|c|c|c|c|c|}
\hline \multirow[t]{2}{*}{ Microbes } & \multirow{2}{*}{$\begin{array}{c}\text { Stroge } \\
\text { time } \\
\text { (month) }\end{array}$} & \multicolumn{4}{|c|}{ Irradiation dose (kGy) } \\
\hline & & 0 & 3 & 5 & 7 \\
\hline \multirow[t]{3}{*}{ TPC } & 0 & $9.45 \times 10^{2}$ & $6.05 \times 10^{2}$ & 0 & 0 \\
\hline & 1 & $1.38 \times 10^{3}$ & $4.37 \times 10^{2}$ & 0 & 0 \\
\hline & 3 & $6.37 \times 10^{3}$ & $3.35 \times 10^{2}$ & 0 & 0 \\
\hline \multirow[t]{3}{*}{ TMYC } & 0 & $2.45 \times 10^{2}$ & $1.71 \times 10^{2}$ & 0 & 0 \\
\hline & 1 & $1.56 \times 10^{3}$ & $1.60 \times 10^{2}$ & 0 & 0 \\
\hline & 3 & $2.54 \times 10^{3}$ & $1.40 \times 10^{2}$ & 0 & 0 \\
\hline \multirow[t]{3}{*}{ E.coli } & 0 & negative & negative & negative & negative \\
\hline & 1 & negative & negative & negative & negative \\
\hline & 3 & negative & negative & negative & negative \\
\hline \multirow[t]{3}{*}{ Coliform } & 0 & negative & negative & negative & negative \\
\hline & 1 & negative & negative & negative & negative \\
\hline & 3 & negative & negative & negative & negative \\
\hline \multirow{3}{*}{$\begin{array}{l}\text { Pseudomonas } \\
\text { spp. }\end{array}$} & 0 & 0 & 0 & 0 & 0 \\
\hline & 1 & 0 & 0 & 0 & 0 \\
\hline & 3 & 0 & 0 & 0 & 0 \\
\hline \multirow{3}{*}{$\begin{array}{l}\text { Slaphylococcus } \\
\text { spp }\end{array}$} & 0 & negative & negative & negative & negative \\
\hline & 1 & negative & negative & negative & negative \\
\hline & 3 & $>10^{3}$ & $>10^{2}$ & negative & negative \\
\hline \multirow{3}{*}{$\begin{array}{l}\text { Clostridium } \\
\text { perfringens }\end{array}$} & 0 & negative & negative & negative & negative \\
\hline & 1 & negative & negative & negative & negative \\
\hline & 3 & negative & negative & negative & negative \\
\hline
\end{tabular}

*Average of three replications

Table 7. Results of some physico-chemical analysis* of spring rolls before and after irradiation at doses up to $7 \mathrm{kGy}$ without storage at $5 \pm 2{ }^{\circ} \mathrm{C}$.

\begin{tabular}{|l|c|c|c|}
\hline \multicolumn{1}{|c|}{ Parameter } & \multicolumn{3}{c|}{ Irradiation dose $(\mathrm{kGy})$} \\
\hline & 0 & 5 & 7 \\
\hline $\mathrm{pH}$ & $4.81 \pm 0.01$ & $4.67 \pm 0.01$ & $4.64 \pm 0.02$ \\
\hline Moisture content $(\%)$ & $72.55 \pm 0.35$ & $75.90 \pm 0.02$ & $72.65 \pm 2.35$ \\
\hline Carbohydrate (\%) & $4.23 \pm 0.18$ & $4.47 \pm 0.02$ & $6.46 \pm 0.09$ \\
\hline Fat $(\%)$ & $3.90 \pm 0.16$ & $2.99 \pm 0.00$ & $3.02 \pm 0.66$ \\
\hline Protein (\%) & $3.64 \pm 0.10$ & $3.45 \pm 0.01$ & $3.76 \pm 0.10$ \\
*Average of three replications
\end{tabular}

Water activity of spring rolls either unirradiated or irradiated samples was in the range of 0.80-0.90. The unirradiated spring roll used during the analysis was ftesh and considered as control. 
Table 8. Results of some physico-chemical analysis* of rissole before and after irradiation at doses up to $7 \mathrm{kGy}$ during storage at $5 \pm 2{ }^{\circ} \mathrm{C}$.

\begin{tabular}{|l|c|c|c|c|c|}
\hline \multirow{2}{*}{ Parameter } & \multirow{2}{*}{$\begin{array}{c}\text { Storage } \\
\text { time } \\
\text { (month) }\end{array}$} & \multicolumn{4}{|c|}{ Irradiation dose (kGy) } \\
\cline { 2 - 6 } & 0 & $0.81 \pm 0.00$ & $0.85 \pm 0.04$ & $0.87 \pm 0.01$ & $0.92 \pm 0.02$ \\
\cline { 2 - 6 } & 3 & $0.86 \pm 0.01$ & $0.86 \pm 0.00$ & $0.80 \pm 0.00$ & $0.90 \pm 0.01$ \\
\hline \multirow{2}{*}{ Aw } & 0 & $7.60 \pm 0.02$ & $7.35 \pm 0.02$ & $7.45 \pm 0.04$ & $7.45 \pm 0.01$ \\
\cline { 2 - 6 } & 3 & $4.21 \pm 0.02$ & $4.40 \pm 0.02$ & $5.30 \pm 0.08$ & $5.90 \pm 0.70$ \\
\hline \multirow{2}{*}{$\begin{array}{l}\text { Moisture } \\
\text { content }(\%)\end{array}$} & 0 & $38.04 \pm 0.29$ & $38.21 \pm 0.22$ & $37.96 \pm 0.02$ & $38.78 \pm 0.21$ \\
\cline { 2 - 6 } $\begin{array}{l}\text { Carbohdrate } \\
(\%)\end{array}$ & 3 & $38.75 \pm 1.00$ & $38.31 \pm 0.89$ & $41.82 \pm 0.19$ & $40.32 \pm 0.75$ \\
\cline { 2 - 6 } & 3 & $16.56 \pm 0.48$ & $17.47 \pm 0.33$ & $16.66 \pm 0.31$ & $16.52 \pm 0.46$ \\
\hline \multirow{2}{*}{ Fat $(\%)$} & 0 & $6.50 \pm 0.36$ & $5.14 \pm 0.25$ & $6.86 \pm 1.01$ & $7.35 \pm 1.11$ \\
\hline \multirow{2}{*}{ Protein $(\%)$} & 3 & $5.16 \pm 0.12$ & $4.60 \pm 0.19$ & $4.07 \pm 0.99$ & $5.22 \pm 0.21$ \\
\cline { 2 - 6 } & 3 & $0.13 \pm 0.00$ & $0.13 \pm 0.01$ & $0.13 \pm 0.00$ & $0.12 \pm 0.01$ \\
\hline
\end{tabular}

*Average of three replications

Table 9. Results of some physico-chemical characteristics* of croquette before and after irradiation at doses up to $7 \mathrm{kG}$ during storage at $5 \pm 2{ }^{\circ} \mathrm{C}$.

\begin{tabular}{|l|c|c|c|c|c|}
\hline \multirow{2}{*}{ Parameter } & \multirow{2}{*}{$\begin{array}{c}\text { Storage } \\
\text { time } \\
\text { (month) }\end{array}$} & 0 & 3 & 5 & 7 \\
\cline { 2 - 6 } & 0 & $0.89 \pm 0.00$ & $0.87 \pm 0.00$ & $0.89 \pm 0.00$ & $0.90 \pm 0.01$ \\
\cline { 2 - 6 } Aw & 3 & $0.88 \pm 0.00$ & $0.88 \pm 0.01$ & $0.86 \pm 0.00$ & $0.85 \pm 0.00$ \\
\hline \multirow{2}{*}{$\mathrm{pH}$} & 0 & $6.63 \pm 0.01$ & $6.64 \pm 0.01$ & $6.65 \pm 0.00$ & $6.66 \pm 0.01$ \\
\cline { 2 - 6 } & 3 & $3.85 \pm 0.02$ & $4.30 \pm 0.03$ & $4.48 \pm 0.02$ & $5.88 \pm 0.09$ \\
\hline \multirow{2}{*}{$\begin{array}{l}\text { Moisture } \\
\text { content }(\%)\end{array}$} & 0 & $36.37 \pm 1.33$ & $39.46 \pm 0.54$ & $39.09 \pm 0.15$ & $39.92 \pm 0.22$ \\
\hline $\begin{array}{l}\text { Carbohydrate } \\
(\%)\end{array}$ & 3 & $40.34 \pm 0.15$ & $40.58 \pm 0.22$ & $41.82 \pm 0.18$ & $39.70 \pm 0.39$ \\
\cline { 2 - 6 } & 3 & $17.79 \pm 0.17$ & $17.73 \pm 0.06$ & $17.56 \pm 0.06$ & $17.45 \pm 0.07$ \\
\hline \multirow{2}{*}{ Fat $(\%)$} & 0 & $10.09 \pm 0.82$ & $7.31 \pm 1.15$ & $8.06 \pm 0.07$ & $9.68 \pm 0.66$ \\
\hline \multirow{2}{*}{ Protein $(\%)$} & 3 & $6.45 \pm 0.35$ & $5.30 \pm 0.25$ & $7.46 \pm 0.25$ & $9.23 \pm 1.03$ \\
\cline { 2 - 6 } & 0 & $0.10 \pm 0.01$ & $0.11 \pm 0.01$ & $0.12 \pm 0.00$ & $0.12 \pm 0.11$ \\
\hline
\end{tabular}

*Average of three replications 
Table 10. Sensoric evaluations* fo unirradiated and irradiated spring rolls, rissole and croquette at doses $5-7 \mathrm{kGy}$ during storage at $5 \pm 2^{\circ} \mathrm{C}$.

\begin{tabular}{|c|c|c|c|c|c|c|c|}
\hline \multirow{2}{*}{$\begin{array}{l}\text { Type of } \\
\text { Snack }\end{array}$} & \multirow{2}{*}{$\begin{array}{l}\text { Irradiation } \\
\text { Dose (kGy) }\end{array}$} & \multirow{2}{*}{$\begin{array}{l}\text { Storage } \\
\text { Time } \\
\text { (month) }\end{array}$} & \multicolumn{5}{|c|}{ Parameter } \\
\hline & & & $\begin{array}{l}\text { General } \\
\text { appearance }\end{array}$ & Colour & Texture & Aroma & Taste \\
\hline \multirow{6}{*}{$\begin{array}{l}\text { Spring } \\
\text { rolls }\end{array}$} & 0 & \multirow[t]{3}{*}{0} & 2.50 & 3.33 & 2.83 & 2.33 & 3.00 \\
\hline & 5 & & 3.75 & 3.92 & 3.75 & 4.08 & 4.60 \\
\hline & 7 & & 3.25 & 3.67 & 3.50 & 2.75 & 3.40 \\
\hline & 0 & \multirow[t]{3}{*}{1} & 1.50 & 2.50 & 1.00 & 1.00 & 1.00 \\
\hline & 5 & & 2.25 & 2.75 & 2.00 & 2.00 & 1.90 \\
\hline & 7 & & 3.75 & 3.50 & 3.30 & 2.75 & 2.40 \\
\hline \multirow[t]{6}{*}{ Rissole } & 0 & \multirow[t]{3}{*}{0} & 3.50 & 5.00 & 3.50 & 4.33 & 3.00 \\
\hline & 5 & & 4.00 & 3.14 & 3.75 & 5.00 & 4.00 \\
\hline & 7 & & 4.00 & 3.14 & 3.00 & 5.00 & 4.00 \\
\hline & 0 & \multirow[t]{3}{*}{3} & 2.75 & 3.50 & 2.50 & 1.25 & 2.75 \\
\hline & 5 & & 3.25 & 3.00 & 3.00 & 3.25 & 3.00 \\
\hline & 7 & & 3.25 & 3.00 & 2.95 & 3.75 & 3.25 \\
\hline \multirow[t]{6}{*}{ Croquette } & 0 & \multirow[t]{3}{*}{0} & 3.50 & 5.00 & 4.00 & 4.33 & 3.60 \\
\hline & 5 & & 3.14 & 4.00 & 3.35 & 4.66 & 4.00 \\
\hline & 7 & & 3.14 & 3.66 & 4.00 & 5.00 & 3.80 \\
\hline & 0 & \multirow[t]{3}{*}{3} & 3.00 & 3.50 & 3.00 & 1.25 & 2.75 \\
\hline & 5 & & 3.14 & 4.00 & 3.00 & 3.25 & 3.25 \\
\hline & 7 & & 3.00 & 3.00 & 4.00 & 4.50 & 3.00 \\
\hline
\end{tabular}

*Average of 10 panelists

It is indicated from Table 9 that panelists could still accept texture, colour and general appearance of irradiated up to 3 months of storage at $5 \pm 2{ }^{\circ} \mathrm{C}$. It seemed that packaging material played an important role in order to maintain the quality, Moreover, some samples sticked to the inner layer and it was difficult to remove it. It is suggested that the intermediate package as well as PET-fray as direct contact packing material is needed. Polypropilene was not suitable for such purpose because the tray was not able to withstand at $-79^{\circ} \mathrm{C}$.

\section{CONCLUSIONS}

It can be concluded from the obtained results that gamma irradiation at the dose of $7 \mathrm{kGy}$ could maintain the quality of spring rolls vacuum packed in a laminate pouch of PET/Alu foil/LLDPE but the best storage time was up to 1 month only. Both rissole and croquette irradiated at doses 5-7 kGy could be kept at $5 \pm 2^{\circ} \mathrm{C}$ for 3 months without affecting their general quality significantly. The use of direct contact and rigid packing material inside the laminate pouch is needed in order to protect damage caused by Vacuum treatment. 


\section{ACKNOWLEDGEMENT}

The authors wish to thank The International Atomic Energy Agency (IAEA) for partly funding this research work. We would also like to express our sincere gratitude to Dr. W.Tatiana Rubio Cabello for her never ending support and encouragement. Special thank addresses to my colleagues Deudeu Lasmawati and Wide Oktorina for their sincere assisstances.

\section{REFERENCES}

1. SENG, C.J., For your convenience, Journal of Asia Pacific Food Industry, October, vol. 15, 9 (2003) p.31-35.

2. IRAWATI, Z., NATALIA, L., NURCAHYA, C.M. and ANAS, F., Irradiation to ensure the safety and quality of home style frozen foods : 1. Liquid base materials, Proceedings of National Seminar XII: "Chemistry in Industry and the Environment", Santika Hotel, Yogyakarta, Indonesia 16-17 December (2003) p.240-252.

3. IRAWATI. Z, MAHA, M., ANSORI, N., NURCAHYA, C.M. and ANAS, F., "Development of shelf-stable foods fish pepes, chicken and meat dishes through radiation processing", Radiation processing for safe, shelf-stable and ready to eat food, Proceedings of a final Research Co-ordination Meeting held in Montreal, Canada, 10-14 July 2000, IAEA-TECDOC-1337, International Atomic Energy Agency, Vienna, Austria (2003) p. 85-99.

4. IRAWATI, Z., NATALIA, N., ANSORI, N., NURCAHYA, C.M., ANAS, F.and SYAFARUDIN, M., "Inoculation packed studies on the shelf-stable food products: I. Effects of gamma irradiation at $45 \mathrm{kGy}$ on the survival of Clostridium sporogenes spores in the foods (preliminary results)", Proceedings of "Qualty in Food Analysis Strengthening the South-East Asian Region", presented at the 5-th Asia Pacific Food Analysis Network Conference, Yogyakarta, 5-7 November 2001, The Asia Pacific Food Analysis Network and Indonesia Institute of Sciences, Indonesia (2001) p. 336·344.

5. MURDIATI, T.B. and BAHRI, S., Food Safety on animal products, Proceedings of "Quality in Food Analysis Strengthening the South-East Asian Region", presented at the 5.th Asia Pacific Food Analysis Network Conference, Yogyakarta, 5-7 November 2001, The Asia Pacific Food Analysis Network and Indonesia Institute of Sciences, Indonesia (2001) p.205 - 216. 
6. PRIADI. A., POERNOMO, S. and NATALIA, L., Presence and level of Salmonella. Coliforms, Escherichia coli, Clostridium perfringens and total aerobic bacteria recovered trom chicken and chicken products in west Java, Indonesia, Proceedings of "Quality in Food Analysis Strengthening the South-East Asian Region", presented at the 5-th Asia Pacific Food Analysis Network Conference, Yogyakarta, 5-7 November 2001, The Asia Pacific Food Analysis Network and Indonesia Institute of Sciences, Indonesia (2001) p.217-224.

7. ASHARI, HADI, UP. and ILHAM, N., Meatballs as the major contribution of beef consumption in Indonesia, Proceedings of "Quality in Food Analysis Strengthening the South-East Asian Region", presented at the 5-th Asia Pacific Food Analysis Network Conference, Yogyakarta, 5-7 November 2001, The Asia Pacific Food Analysis Network and Indonesia Institute of Sciences, Indonesia (2001) p.231-246. 